\title{
DESIGN AND OPERATION OF SYNCHRONIZED ROBOTIC ARM
}

\author{
Goldy Katal ${ }^{1}$, Saahil Gupta ${ }^{2}$, Shitij Kakkar ${ }^{3}$ \\ ${ }^{1}$ Student, Electrical and Electronics Department, Maharaja Agrasen Institute of Technology, Delhi, India, \\ ${ }^{2,3}$ Student, Electrical and Electronics Department, HMR Institute of Technology, Delhi, India, \\ katal.goldy@gmail.com, saahilgpt005@gmail.com, shitijkakkar91@gmail.com
}

\begin{abstract}
The paper manuscript deals with the designing and implementation of Synchronized Robotic Arm, which is used to perform all the basic activities like picking up objects and placing them. In this paper, a robotic arm is designed, synchronized with the working arm and would perform the task as the working arm does. The work done by the robotic arm would be highly precise, as a digital servo motor is used. A servo motor of 230 oz-inch is used in the project, but motors with more capacity can also be used as according to the desired work. This robotic arm can also be used for precision works. For instance some work has to be done very precisely but the conditions do not suit human beings. In such conditions, this robotic arm can be used remotely and the task can be accomplished. The programming is done on ATMEGA-8 Microcontroller using Arduino programming. The potentiometers are also used to detect the angle of rotation and the signals are then sent to the microcontroller. In today's world, this Robotic arm has turned out very benevolent. Besides Robotics and Automation, these kinds of arms have applications in other fields also.
\end{abstract}

Keywords -Arduino, ATMEGA-8, IC, Potentiometers, Servo Motors

_***

\section{INTRODUCTION}

A Robot is a virtually intelligent agent capable of carrying out tasks robotically with the help of some supervision. Practically, a robot is basically an electro-mechanical machine that is guided by means of computer and electronic programming. Robots can be classified as autonomous, semiautonomous and remotely controlled[6].Robots are widely used for variety of tasks such as service stations, cleaning drains, and in tasks that are considered too dangerous to be performed by humans. A robotic arm is a robotic manipulator, usually programmable, with similar functions to a human arm .This Robotic arm is programmable in nature and it can be manipulated. The robotic arm is also sometimes referred to as anthropomorphic as it is very similar to that of a human hand. Humans today do all the tasks involved in the manufacturing industry by themselves. However, a Robotic arm can be used for various tasks such as welding, drilling, spraying and many more[1].A self sufficient robotic arm is fabricated by using components like micro-controllers and motors. This increases their speed of operation and reduces the complexity. It also brings about an increase in productivity which makes it easy to shift to hazardous materials. In the implementation process, the necessary components of structure ICs, blocks and power supply are all assembled on the PCB[9].The main part of the design is ATMEGA-8 micro-controller which coordinates and controls the product's action. This specific micro controller is used in various types of embedded applications. Robotics involves elements of mechanical and electrical engineering, as well as control theory, computing and now artificial intelligence. According to the Robot Institute of America, "A robot is a reprogrammable, multifunctional manipulator designed to move materials, parts, tools or specialized devices through variable programmed motions for the performance of a variety of tasks [9].The robots interact with their environment, which is an important objective in the development of robots[8]. This interaction is commonly established by means of some sort of arm and gripping device or end effectors. In the robotic arm, the arm has a few joints, similar to a human arm, in addition to shoulder, elbow, and wrist, coupled with the finger joints; there are many joints [7].

The design process is clearly explained in the next section with detailed information regarding the components which are used, followed by the implementation leading to results and finally ends with conclusion.

\section{DESIGN OF ROBOTIC ARM}

The Robotic Arm is designed using the Microcontroller i.e. ATMEGA8 Micro-controller using Arduino programming. This process works on the principle of interfacing servos and potentiometers. This task is achieved by using Arduino board. Potentiometers play an important role The remote is fitted with potentiometers and the servos are attached to the body of the robotic arm. The potentiometer converts the mechanical motion into electrical motion. Hence, on the motion of the remote the potentiometers produce the electrical pulses, which are en route for the arduino board. The board then processes the signals received from the potentiometers and finally, converts them into requisite digital pulses that are then sent to the servomotors. This servo will respond with regards to the pulses which results in the moment of the arm [5]. 
Figure 1 shows the image of a servo motor. It consists of a motor which is coupled to a sensor, used for position feedback, through a reduction gearbox. It also accompanies a relatively sophisticated controller, usually a dedicated module designed specifically for use with servomotors In short, the micro controller interfaces all these components specified below. A short list of components include

1. Servo motors

2. Potentiometers

3. ATMEGA 8

4. Figure 1 shows the image of a servo motor. It consists of a motor which is coupled to a sensor, used for position feedback, through a reduction gearbox. It also accompanies a relatively sophisticated controller, usually a dedicated module designed specifically for use with servomotors

In short, the micro controller interfaces all these components specified below. A short list of components include

1. Servo motors

2. Potentiometers

3. ATMEGA 8

4. Arduino Deumilanove

\subsection{Circuit Diagram}

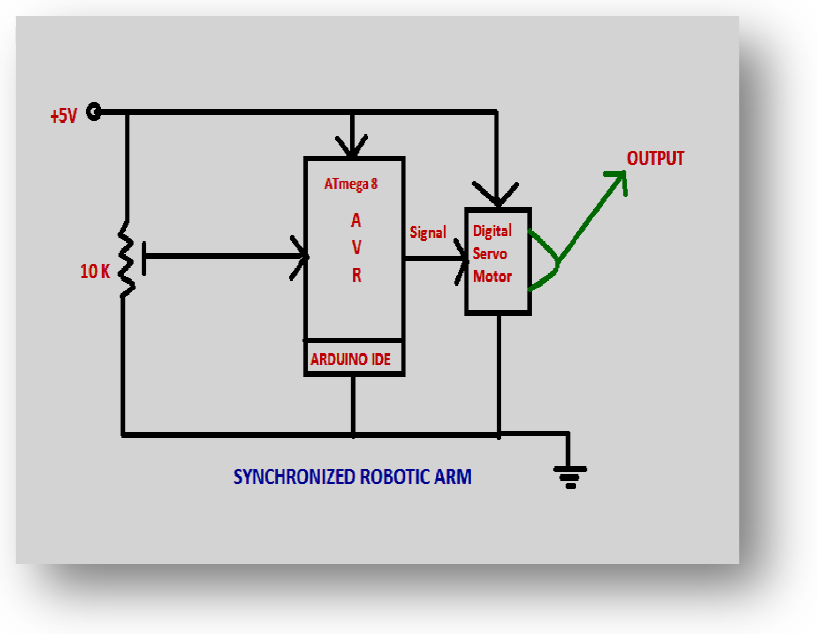

Fig 1: Circuit Diagram of a Robotic Arm

\subsection{Servo motors}

Servo refers to an error sensing feedback control which is used to correct the performance of a system. Servo or RC Servo Motors are DC motors equipped with a servo mechanism for precise control of angular position. The RC servo motors usually have a rotation limit from $90^{\circ}$ to $180^{\circ}$. But servos do not rotate continually. Their rotation is restricted in between the fixed angles. A servomotor is a motor, which forms part of a servomechanism.[12]

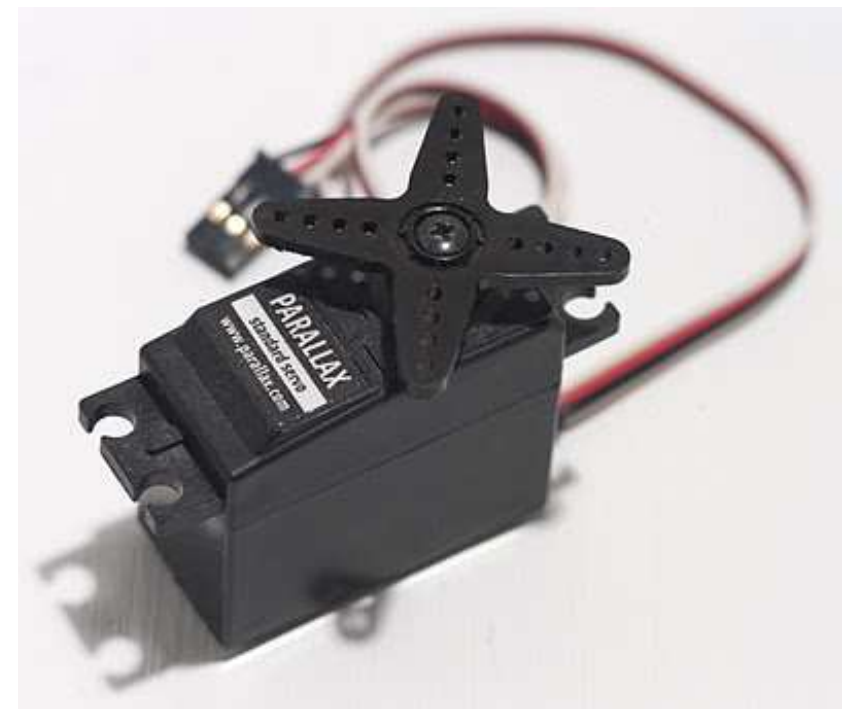

Fig 2: A Servo Motor

The Servos are used for precision positioning. They are used in robotic arms and legs, sensor scanners and in RC toys like RC helicopter, airplanes and cars. The specifications for Servomotor used are as follows:

- Weight- 55g

- Dimension- $40.7 * 19.7 * 42.9 \mathrm{~mm}$

- Stall torque- $10 \mathrm{~kg} / \mathrm{cm}$

- Operating speed- $0.20 \mathrm{sec} / 60$ degree(4.8v)

- Operating voltage 4.8-7.2V.

- Temperature range 0-55 degrees.

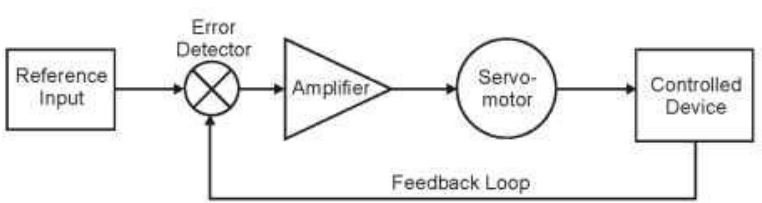

Fig 3: Block diagram of a Servo Motor

\subsection{Potentiometers}

A Potentiometer (pot for shot) is a variable resistor. The two output terminals act as a fixed resistor. A movable contact called a wiper (or a slider) moves across the resistor, producing a variable resistance between the centre terminals and the two sides[12].A potentiometer is basically a voltage divider which is used for the measurement of electric potential. Potentiometers are commonly used to control electrical devices such as volume controls on audio equipment. Potentiometers which are operated by a mechanism can also be used as position transducer. 


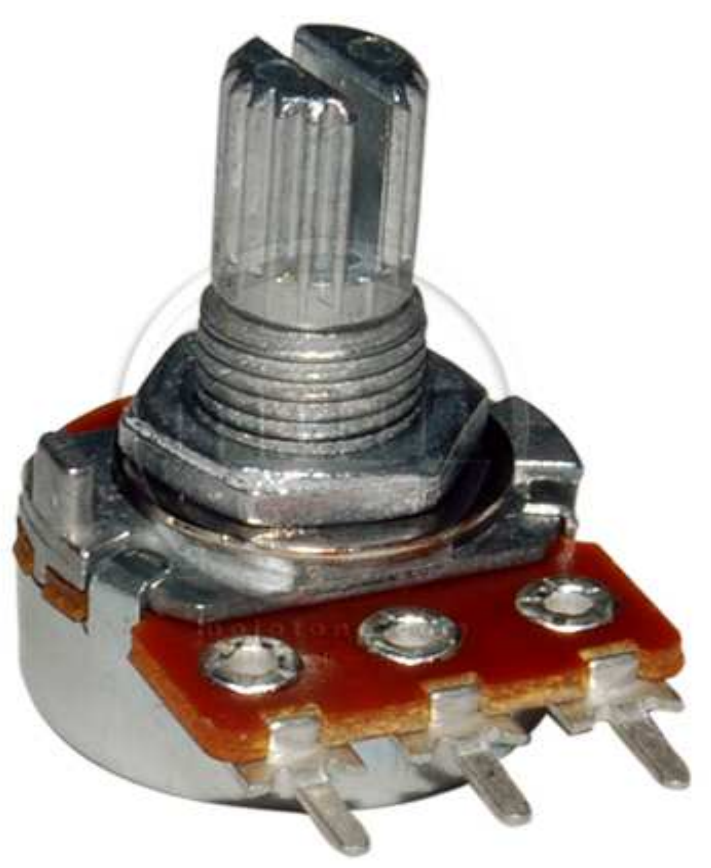

Fig 3: A Potentiometer

A potentiometer is a manually adjustable electrical resistor that uses three terminals. In many electrical devices, potentiometers are establishes the levels of output. For example, in case of a loudspeaker, a potentiometer is used to adjust the volume or taken the case of a television set, computer monitor or light dimmer; it can be used to control the brightness of the screen or light bulb.

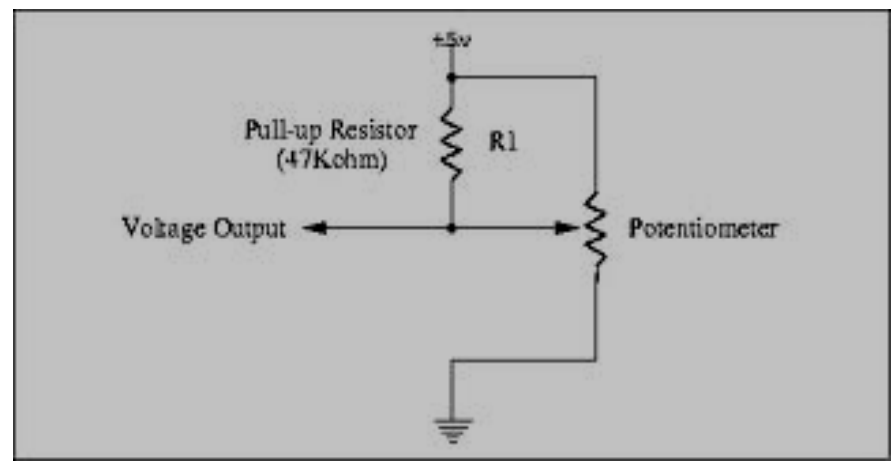

Fig 5: Circuit Diagram of a Potentiometer

\subsection{Arduino Deumilanove}

Arduino is a single-board microcontroller (accepted open source), successor of the open-source wiring platform which is designed to devise the process of using electronics in multidisciplinary functions [10].

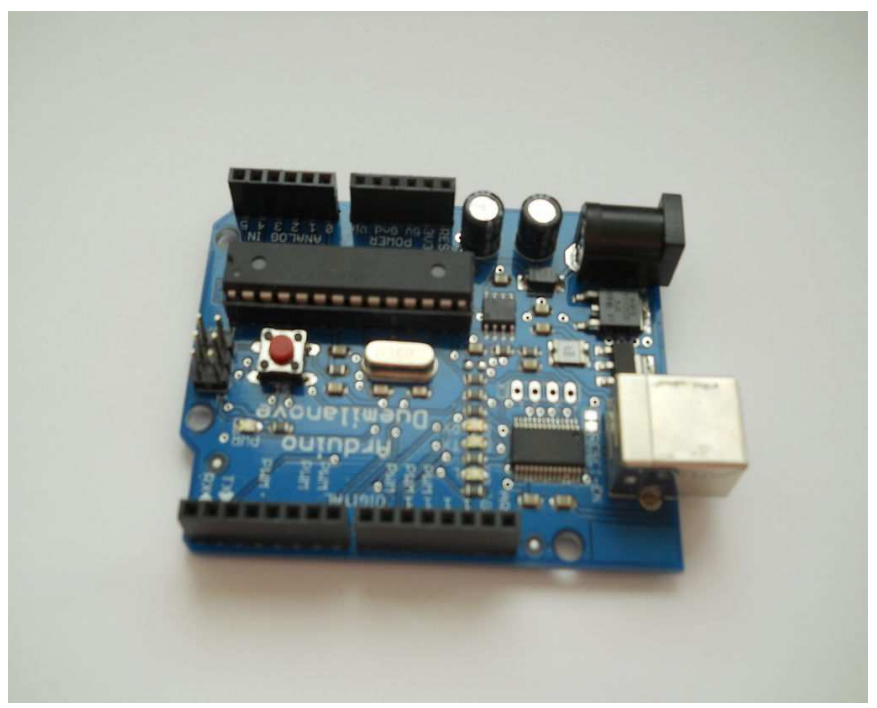

Fig 6: An Arduino Deumilanove

Arduino board consists of the following parts :

- USB connector

- Power connector

- Automatic power switch

- Digital pins

- Analog pins

- Power pins

- Reset switch

\section{OPERATION PROCESS}

\subsection{Working Principle}

The working principle can be understood from the figure 7.If we want the robotic device to undertake a given task, then it is important to control it and it should also possess the capability of sending specific orders to the manipulator which has to be carried out in terms of positions or velocities of its final effectors [2]. Secondly, it should be able to sense the real obtained position or velocity. There are many other sensing capabilities that may be pivotal depending on the application. Therefore, in order to implement a control algorithm for the manipulator to solve a particular task, it is mandatory to control its basic functions which include moving and sensing, which further leads to the development of a software platform [3]. Basically, a robotic manipulator and sensors manufacturers provides a set of functions to interface with the low level features of robots and sensors. Now these functions are needed to be integrated within the full software applications which are then used to solve a particular task. 


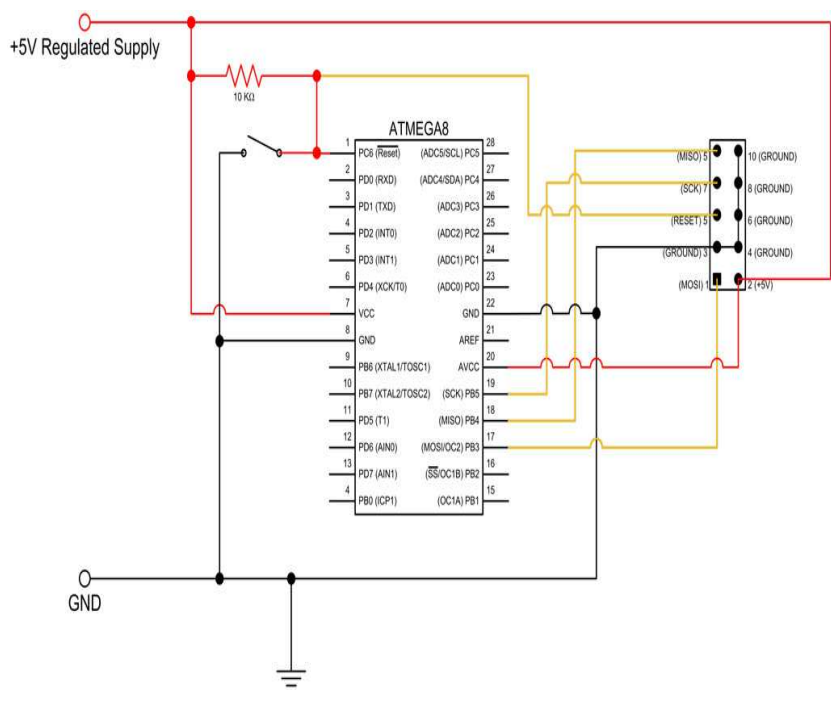

Fig 7: Complete Circuit Block Diagram

\subsection{ATMEGA8 Micro-controller}

ATmega8 is a AVR 8-BIT Microcontroller featuring Insystem Programmable capability along with Flash code storage. This micro-controller is re-programmable up to 1000 times. It also features 32 working registers, single clock cycle execution giving up to $1 \mathrm{MIPs} / \mathrm{MHz}$

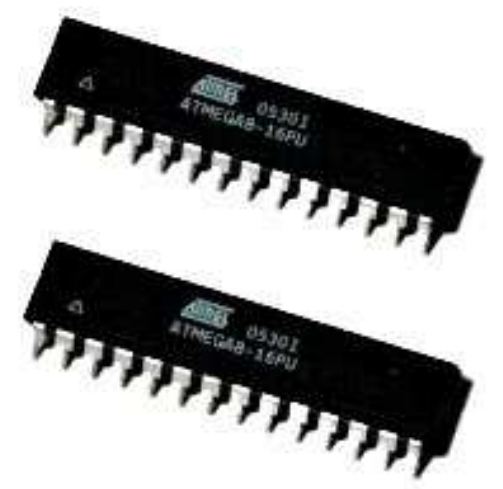

Fig 8: ATMEGA 8

The key features of ATMEGA8 are :

- $\quad$ KK BYTES of In-System Programmable Flash

- 512 Bytes EEPROM, Endurance: 100,000 Write/Erase Cycles

- Two 8-bit Timer/Counters with Separate Prescaler, one Compare Mode

- Analog Comparator

- One 16-bit Timer/Counter with Separate Prescaler, Compare Mode, and Capture Mode

- $\quad$ Real Time Counter with Separate Oscillator
- 6-channel ADC -Four Channels 10-bit Accuracy and Two Channels 8-bit Accuracy

- Programmable Serial USART

- External and Internal Interrupt Sources

- 23 Programmable I/O Lines

- 4.5 to $5.5 \mathrm{~V}$ operation

The low-power Atmel 8-bit AVR RISC-based microcontroller consists of $8 \mathrm{~KB}$ of programmable flash memory, $1 \mathrm{~KB}$ of SRAM, 512K EEPROM, and a 6 or 8 channel 10-bit A/D converter. The device supports throughput of 16 MIPS at 16 $\mathrm{MHz}$ and operates between 2.7-5.5volts.

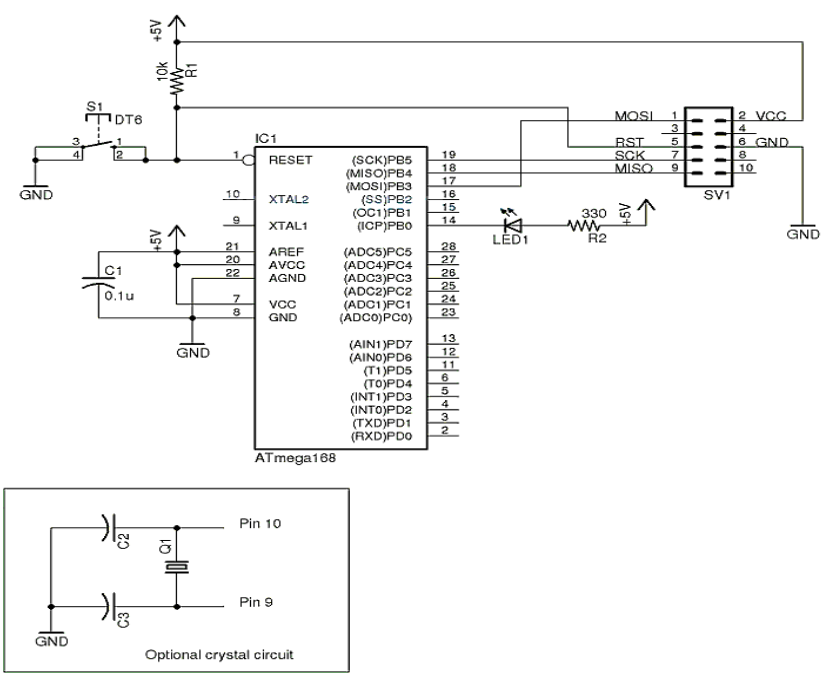

Fig 9: Schematic view of Atmel AVR

\section{APPLICATIONS OF ROBOTIC ARM}

- The robotic arm can be designed to perform any desired task such as welding, gripping, spinning etc., depending on the application. For example robot arms in automotive assembly line perform a variety of tasks such as wielding and parts rotation and placement during assembly.

- In space the space shuttle Remote Manipulator System have multi degree of freedom robotic arms that have been used to perform a variety of tasks such as inspections of the Space Shuttle using a specially deployed boom with cameras and sensors attached at the end effectors.

- The robot arms can be autonomous or controlled manually and can be used to perform a variety of tasks with great accuracy. The robotic arm can be fixed or mobile (i.e. wheeled) and can be designed for industrial or home applications. Robotic hands often have built-in pressure sensors that tell the computer how hard the robot is gripping a particular object. This keeps the robot from dropping or breaking whatever it's carrying. 
Other end effectors include blowtorches, drills and spray painters. this improves their performance.

- In medical science: "Neuroarm" uses miniaturized tools such as laser scalpels with pinpoint accuracy and it can also perform soft tissue manipulation, needle insertion, suturing, and cauterization.

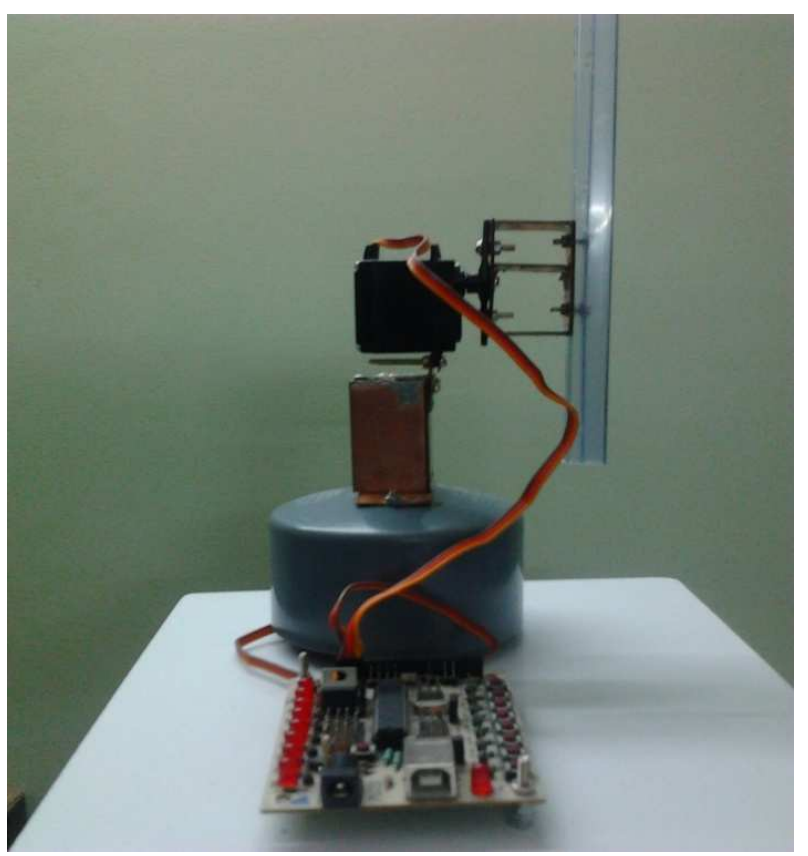

Fig 10: A Robotic Arm

\section{FUTURE WORK}

The future scope of Robotic Arm is commendable. It will involve patients who are paralyzed from down the neck can manipulate robotic arms with their thoughts. It will be able to direct a mechanical arm to pick up a bottle of coffee and bring it to her lips. It was the first time she'd been able to drink without assistance in nearly 15 years. There will be a thriving need for the Robotic arm in military, industrial and many more.

\section{REFERENCES}

[1] C. F. Olson, Lab. JP, Technol. CIo, Pasadena, "Probabilistic self localization for mobile robots"; IEEE Transactions on Robotics and Automation 16,1, 55-66, 2000.

[2] R.B. Gillespie, J. E. Colgate, M. A. Peshkin, "A general framework for robot control"; IEEE Transactions on Robotics and Automation, 17,4, 391-401, 2001.

[3] Devendra P. Garg and Manish Kumar, "Optimization Techniques applied to multiple manipulators for path planning and torque minimization"; Engineering Applications of Artificial Intelligence 15, 3-4, 241-252, 2002.

[4] J. C. Trinkle and R. James Milgram, "Complete Path Planning for Closed Kinematics Chains with Spherical Joints";
SAGE International Journal of Robotic Research 21, 9, 773789, 2002.

[5] M. Gemeinder and M. Gerke, "GA-based Path Planning for Mobile Robot Systems employing an active Search Algorithm"; Journal of Applied Soft Computing 3, 2, 149-158, 2003. [2]. "Six-servo Robot Arm" from www.arexx.com.cn on 13-11-2011 pg: 1-18

[6]. Jegede Olawale, Awodele Oludele, Ajayi Ayodele, "Development of a Microcontroller Based Robotic Arm", in Proceedings of the 2007 Computer Science and IT Education Conference pg: 549-557.

[7]. "Six-servo Robot Arm" from www.arexx.com.cn on 1311-2011.

[8]. "Robotic Arm" - www. NASA explores. com

[9]. "Robot software" from Wikipedia, the free encyclopedia on 04-3-2012

[10]. "ATMEL - short notes", www.atmel.com

[11]. "Arduino MCU", - www.arduino.com

[12]. "Servomotors \& system design components", www.bhashaelectronics. Com 